\title{
Early-type variables in the Magellanic Clouds
}

\section{I. $\beta$ Cephei stars in the LMC bar}

\author{
A. Pigulski and Z. Kołaczkowski
}

Wrocław University Observatory, Kopernika 11, 51-622 Wrocław, Poland

Received 13 February 2002 / Accepted 21 March 2002

\begin{abstract}
A thorough analysis of the OGLE-II time-series photometry of the Large Magellanic Cloud bar supplemented by similar data from the MACHO database led us to the discovery of three $\beta$ Cephei-type stars. These are the first known extragalactic $\beta$ Cephei-type stars. Two of the three stars are multiperiodic. Two stars have inferred masses of about $10 M_{\odot}$ while the third is about 2 mag brighter and at least twice as massive. All three variables are located in or very close to the massive and young LMC associations (LH 41, 59 and 81). It is therefore very probable that the variables have higher than average metallicities. This would reconcile our finding with theoretical predictions of the shape and location of the $\beta$ Cephei instability strip in the H-R diagram. The low number of $\beta$ Cephei stars found in the LMC is another observational confirmation of strong dependence of the mechanism driving pulsations in these variables on metallicity. Follow-up spectroscopic determination of the metallicities in the discovered variables will provide a good test for the theory of pulsational stability in massive main-sequence stars.
\end{abstract}

Key words. stars: early-type - stars: oscillations - stars: variable: other - stars: abundances - Magellanic Clouds

\section{Introduction}

Since the early $90 \mathrm{~s}$ it is known that pulsations in $\beta$ Cephei stars are driven by the $\kappa$ mechanism operating at a temperature around $2 \times 10^{5} \mathrm{~K}$ where the opacity bump occurs (Cox et al. 1992; Moskalik \& Dziembowski 1992; Kiriakidis et al. 1992; Dziembowski \& Pamyatnykh 1993; Gautschy \& Saio 1993). The bump is caused by a large number of bound-bound transitions in the iron-group ions. As a consequence, the area of the instability strip in the Hertzsprung-Russell (H-R) diagram should strongly depend on metallicity (Moskalik \& Dziembowski 1992; Pamyatnykh 1999). The instability in models of $\beta$ Cephei stars results from a small domination of driving over damping (see, e.g., Moskalik \& Dziembowski 1992), thus the subtle differences in both the input physics and the modeling may lead to considerable differences in the predicted position of the instability strip. For instance, for solar metallicity, Pamyatnykh (1999) finds that the $\beta$ Cephei instability strip (hereafter BCIS) has no upper edge for the most massive stars and extends beyond the corehydrogen burning phase of evolution. Moreover, for the heavy elements abundance parameter $Z=0.01$, his BCIS shrinks down to a small region at high luminosities, where $\beta$ Cephei stars have not been found. On the other hand, for

Send offprint requests to: A. Pigulski,

e-mail: pigulski@astro.uni.wroc.pl solar abundance, Deng \& Xiong (2001) predict the much narrower BCIS with an upper boundary for $M \approx 20 M_{\odot}$. In their calculations the BCIS disappears at $Z$ as low as 0.005 .

In this context, the observational study of $\beta$ Cephei star pulsations in objects of different metallicity is of great importance. It was already pointed out by Sterken \& Jerzykiewicz (1988) that with their lower than Galactic metallicities and relatively small interstellar absorption, the Large and Small Magellanic Clouds (LMC and SMC) are among the best objects for such a study. Although typical photometric amplitudes of $\beta$ Cephei stars are of the order of $0.01 \mathrm{mag}$ and these stars should not be brighter than $\sim 14 \mathrm{mag}$ in the LMC and even fainter in the SMC, with the modern techniques allowing CCD photometry in crowded fields, the detection of these stars is certainly within reach.

Searches for $\beta$ Cephei stars in the Magellanic Clouds had already been undertaken. The first was carried out by Sterken \& Jerzykiewicz (1988). By means of the photoelectric photometry these authors studied six late O/early B-type stars. For the same purpose, Kubiak (1990) searched the young LMC cluster NGC 1712. These searches resulted in the discovery of some variables, but none of a convincing $\beta$ Cephei-type pulsation mainly because of the small statistical sample observed. Another search for $\beta$ Cephei stars was performed by 
Balona (1992, 1993) and Balona \& Jerzykiewicz (1993) in NGC 2004 and 2100 in the LMC and in NGC 330 in the SMC. A similar CCD search was also carried out by Kjeldsen \& Baade (1994) in NGC 2122 in the LMC and NGC 371 in the SMC. No variable of $\beta$ Cephei-type was found by these authors.

The reduction of the OGLE-II data (Udalski et al. 1997) for the Magellanic Clouds by means of the Difference Image Analysis (hereafter DIA, Woźniak 2000; Żebruń et al. 2001a) yielded the photometry for over 53000 candidate variables in the LMC and 15000 in the SMC. A catalog including these stars was recently made available to the astronomical community (Żebrun et al. 2001b). Earlier, the OGLE $B V I_{\mathrm{C}}$ photometry of about $6.7 \times 10^{6}$ stars in the LMC (Udalski et al. 2000) and $2.2 \times 10^{6}$ stars in the SMC (Udalski et al. 1998) was published. At the same time, the even larger database of the MACHO observations of $7.3 \times 10^{7}$ stars in both Clouds and in two filters $(V, R)$ has become available (Allsman \& Axelrod 2001).

These databases offer an unprecedented opportunity to search for the presence of $\beta$ Cephei-type stars in the LMC and SMC. Combined with the detailed metallicity determinations, this result will put strong constraints on the theoretical stability predictions. As we shall show later, the accuracy of the photometry is good enough to detect all $\beta$ Cephei-type pulsations in the LMC stars with semiamplitudes exceeding 3 to $5 \mathrm{mmag}$, depending on the star brightness.

In this paper, intended to be the first of a series, we present the results of the search for $\beta$ Cephei stars among the LMC bar stars using the OGLE-II DIA photometry. A similar analysis for SMC stars will be published separately. The details of the analysis and the selection of stars is described in Sect. 2. Section 3 presents the main results of the search. The possible connection of the variables we found with the LMC clusters and associations is verified in Sect. 4. We also briefly discuss the variables detected during previous attempts to find $\beta$ Cephei stars in the LMC (Sect. 5). The consequences of our finding are discussed in detail in Sect. 6, while a short summary and our plans concerning the next papers of the series are given in the last section.

\section{Data selection and analysis}

The output of the DIA is the photometry of the variable stars only (strictly speaking the candidates for variable stars, as the DIA produces also artifacts), therefore it was an obvious choice to take the OGLE-II catalog of about 53400 candidate variables in the LMC (Żebruń et al. 2001b) as the main source of the time-series photometric data. For the details of the OGLE-II observations and the DIA reductions we direct the reader to the paper of Żebruń et al. (2001a). We only mention that the OGLE-II observations of the LMC cover twenty-one $14 ! 2 \times 56 ! 9$ fields in the bar of this galaxy. For a single star, about 30,40 , and 400 observations are typically available

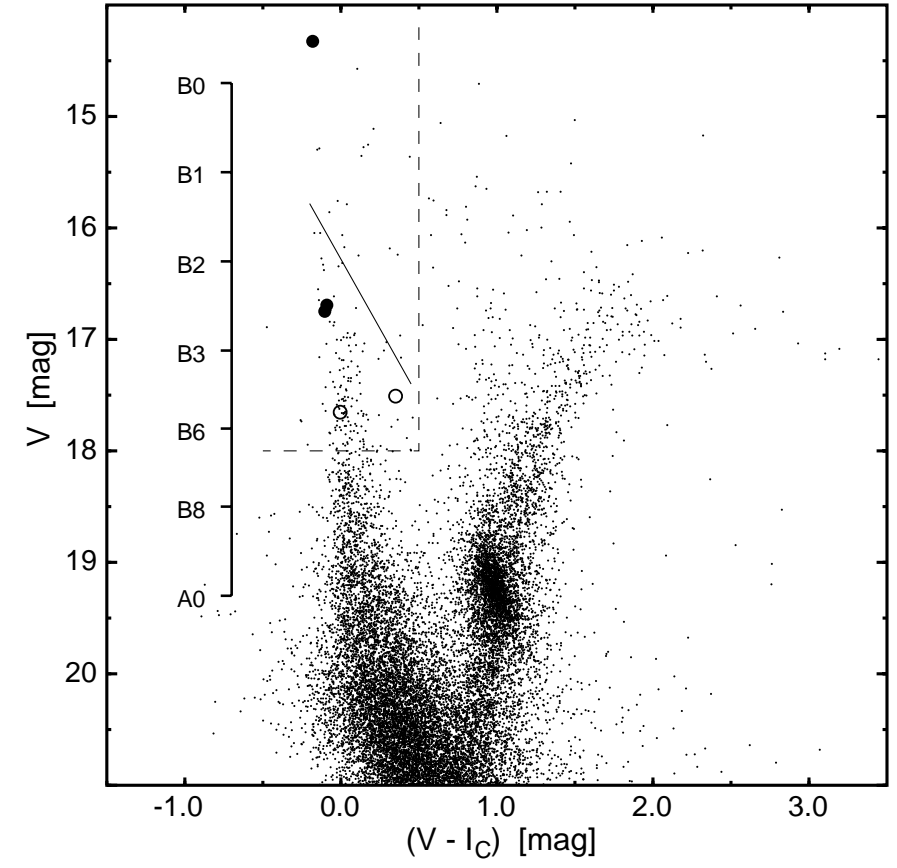

Fig. 1. Colour-magnitude diagram for $\sim 16000$ stars in the OGLE-II LMC field SC1, randomly selected from the photometry of Udalski et al. (2000). The three $\beta$ Cephei stars, $\mathrm{V} 1, \mathrm{~V} 2$, and V3, are shown as filled circles, the other two short-period variables, V4 and V5, as open circles. For reference, the location of the luminosity class V B-type stars is shown considering an LMC distance modulus of $18.5 \mathrm{mag}$ and $A_{V}=0.4 \mathrm{mag}$. The inclined solid line is the reddening line for $A_{V} / E\left(V-I_{\mathrm{C}}\right)=2.49$ (Stanek 1996). The dashed line shows the limits of the search for $\beta$ Cephei variables.

in the $B, V$ and $I_{\mathrm{C}}$ bands, respectively. The OGLE-II observations span at most $3.5 \mathrm{yr}$, from the beginning of 1997 to mid-2000.

The MACHO data archive (Allsman \& Axelrod 2001) served as the second source for the time-series data. The data span about 8 years between mid-1992 and the beginning of 2000, and are available in two passbands: blue $(440-590 \mathrm{~nm})$ and red $(590-780 \mathrm{~nm})$. Henceforth we shall refer to these passbands as $V_{\mathrm{M}}$ and $R_{\mathrm{M}}$, respectively. The MACHO observations cover a much larger area around the LMC than the OGLE-II ones do. However, the database is at present accessible in an unsuitable way for our search. We therefore use here the MACHO archive only for stars that we found particularly interesting from the analysis of the OGLE-II data and for variables detected in previous searches (see Sect. 5). A thorough search for $\beta$ Cephei stars in the MACHO archive will be done as soon as enhanced search capabilities become available.

Prior to the analysis, the observations with larger photometric errors have been rejected from the MACHO data. In addition, some outliers were removed. Finally, the heliocentric corrections were applied to the MACHO epochs. Since the published epochs correspond to the beginning of an exposure (Cook 2002), 150 s correction (half the exposure time) was added to them. The OGLE-II epochs have 
been corrected for the "drift-scan effect" according to the equation given by Żebruń et al. (2001b).

The coldest and faintest Galactic $\beta$ Cephei stars have MK type of about $\mathrm{B} 2.5 \mathrm{~V}$, corresponding to $M_{V} \approx$ -2 . Taking into account the LMC distance modulus of $18.5 \mathrm{mag}$, we get $V=16.5 \mathrm{mag}$. The average $E(B-V)$ colour excess for the LMC is about 0.13 mag (Massey et al. 1995), yielding $A_{V} \approx 0.4 \mathrm{mag}$. However, the visual extinction is sometimes much larger, especially for hot stars (Zaritsky 1999). This is obvious, because there is usually a large amount of interstellar matter around young hot objects. In order to take that into account, we decided to add a 1 mag margin to the limiting magnitude of our search. Considering also the effect of reddening on the photometric indices, we have finally chosen stars with $V<18 \mathrm{mag}$ and $\left(V-I_{\mathrm{C}}\right)<0.5 \mathrm{mag}$ (see Fig. 1) to search for the presence of $\beta$ Cephei-type variability. A total of 5204 stars in the LMC were extracted from the catalogue of Żebruń et al. (2001b).

For all these stars we performed frequency analysis by means of the AoV periodogram of Schwarzenberg-Czerny (1996) in the range between 0 and $20 \mathrm{~d}^{-1}$. The frequencies of the highest peaks in all periodograms were then sorted and the photometry of stars with the highest frequencies was examined visually.

In the classical approach, $\beta$ Cephei stars are recognized as early B-type stars with periods shorter than $0.3 \mathrm{~d}$ (Sterken \& Jerzykiewicz 1993). Since it seems that $\beta$ Cephei stars with periods slightly longer than $0.3 \mathrm{~d}$ exist (Krzesiński \& Pigulski 1997), we decided to consider all stars showing periods shorter than $0.35 \mathrm{~d}$ as potential variables of this type.

As a result of our periodogram analysis, we found three stars which we regard to be the LMC $\beta$ Cephei stars. Two other short-period objects were found among early-type variables. They could also be $\beta$ Cephei stars, but because another interpretation of their variability is more likely, we describe them only briefly in Sect. 3.4.

For the sake of simplicity we designate the $\beta$ Cephei stars as V1 to V3 and list them in Table 1. The crossidentifications with the OGLE-II and MACHO designations are also given. The finding charts shown in Fig. 2 were taken from the OGLE-II database.

\section{3. $\boldsymbol{\beta}$ Cephei stars}

\section{1. $V 1=O G L E 053446.82-694209.8=$ MACHO 81.8881.161}

The star is located in the field SC1 and 81 of the OGLE-II and MACHO, respectively. Fourier analysis of the OGLE-II $I_{\mathrm{C}}$ observations of this star (Fig. 3) revealed a single frequency $f=4.05160 \mathrm{~d}^{-1}$. No other periodicity above the noise level $(\sim 5 \mathrm{mmag})$ has been found. Practically the same result, that is, a monoperiodic signal, has been detected in the MACHO data except that after prewhitening with $f$, a small peak appeared at a frequency of 1 (sidereal day) $)^{-1}$. It is presumably of
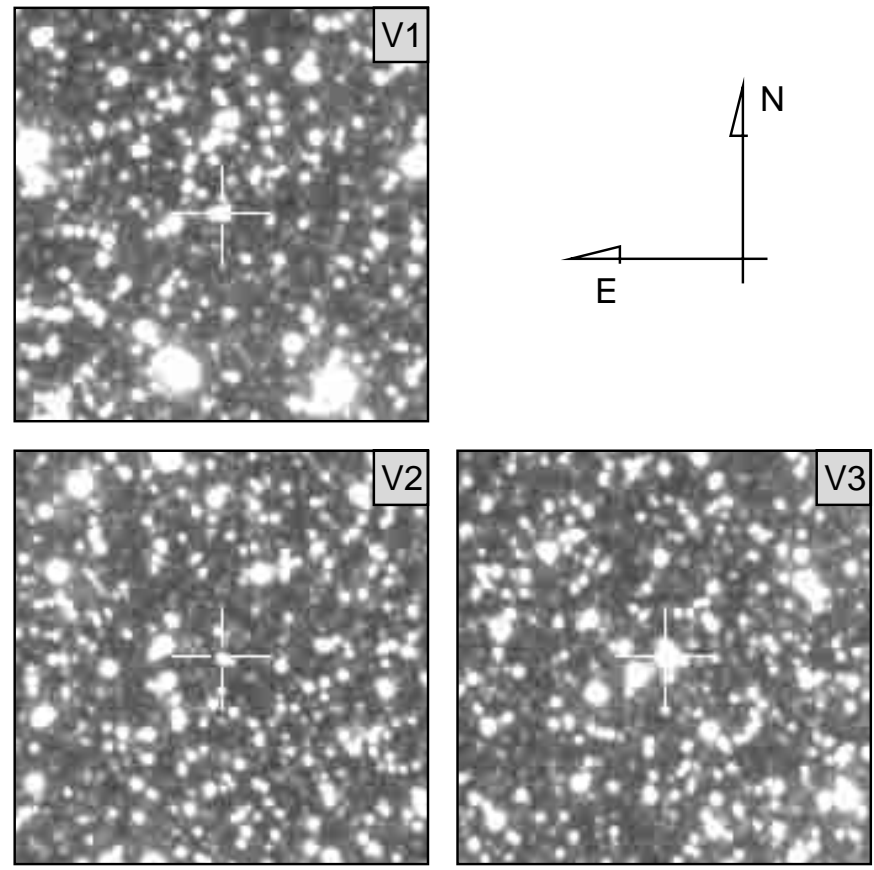

Fig. 2. OGLE-II maps $\left(1{ }^{\prime} 17 \times 11^{\prime} 17\right)$ for the three $\beta$ Cephei stars (V1-V3) in the LMC bar. The J2000 coordinates of the stars are coded within the OGLE-II names (Table 1).

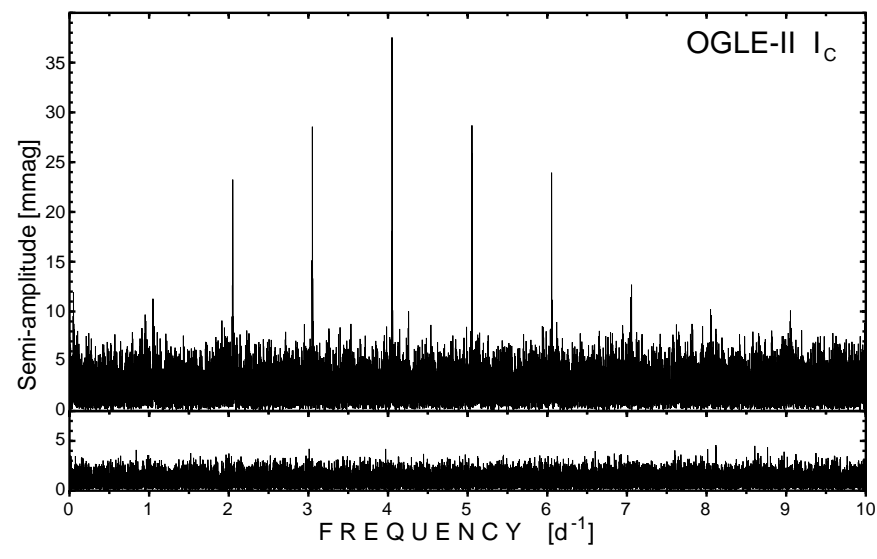

Fig. 3. Fourier periodograms of the OGLE-II 1997-2000 $I_{\mathrm{C}-}$ filter observations of V1. The upper panel shows the periodogram of the original data; the lower one, after prewhitening with $f=4.05160 \mathrm{~d}^{-1}$.

instrumental origin. The star has virtually the same amplitudes in the $V_{\mathrm{M}}$ and $R_{\mathrm{M}}$ bands (see Table 2). Since the MACHO data span a wider time interval than the OGLEII ones do, the refined frequency, $f=4.051593 \mathrm{~d}^{-1}$, has been obtained by means of a non-linear least squares fitting of the MACHO data. The semi-amplitudes and phases of both datasets are given in Table 2 .

\section{2. $V 2=$ OGLE 052809.21-694432.1 = MACHO 77.7792.493}

The second $\beta$ Cephei-type variable has been found in the OGLE-II field SC3 and in the MACHO field 77. It has almost the same colour and magnitude as V1 (see Fig. 1 
Table 1. The $\beta$ Cephei stars in the LMC bar.

\begin{tabular}{ccccccc}
\hline \hline Name & $\begin{array}{c}\text { OGLE-II } \\
\text { field }\end{array}$ & $\begin{array}{c}\text { OGLE-II } \\
\text { name }\end{array}$ & $\begin{array}{c}\text { MACHO } \\
\text { name }\end{array}$ & $\begin{array}{c}V \\
{[\mathrm{mag}]}\end{array}$ & $\begin{array}{c}B-V \\
{[\mathrm{mag}]}\end{array}$ & $\begin{array}{c}V-I_{\mathrm{C}} \\
{[\mathrm{mag}]}\end{array}$ \\
\hline V1 & LMC-SC1 & OGLE053446.82-694209.8 & 81.8881 .161 & 16.691 & -0.109 & -0.089 \\
V2 & LMC-SC3 & OGLE052809.21-694432.1 & 77.7792 .493 & 16.748 & +0.003 & -0.103 \\
V3 & LMC-SC7 & OGLE051841.98-691051.9 & - & 14.327 & -0.175 & -0.181 \\
\hline
\end{tabular}

and Table 1). The Fourier periodogram of the OGLE-II $I_{\mathrm{C}}$-filter data (Fig. $4 \mathrm{a}$ ) shows the strongest signal at frequency $f_{1}=3.49767 \mathrm{~d}^{-1}$. After removing this signal the highest peak appears at a frequency $f_{\mathrm{sd}}=1.00333 \mathrm{~d}^{-1}$,

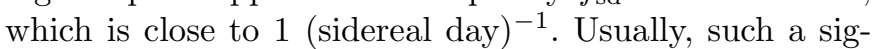
nal appears in the periodogram when long-term irregular changes are superimposed. Since it appears for V2 in all three datasets, it can be caused by a low-frequency intrinsic variation. In fact, changes in the mean magnitude of this star are well seen in the MACHO data: during the first three years of observations the mean brightness of V2 faded by about $0.02 \mathrm{mag}$. After removing $f_{1}$ and the lowfrequency signals, a second periodicity, $f_{2}=3.68377 \mathrm{~d}^{-1}$, with a very small amplitude appears slightly above the noise level (Fig. 4a). In order to make sure this is a real frequency, we calculated similar periodograms for the MACHO data (Fig. 4b). Although barely visible in the $V_{\mathrm{M}}$ observations, $f_{2}$ appears clearly in the $R_{\mathrm{M}}$ data. We conclude that the frequency is real, and thus V2 is a biperiodic variable. However, because of the low amplitude of this periodic signal, a few close peaks of similar height occur around $f_{2}$. The highest peak does not have the same frequency in all periodograms; it is equal to $3.6838,3.6853$, and $3.6847 \mathrm{~d}^{-1}$ for the OGLE-II $I_{\mathrm{C}}$, MACHO $V_{\mathrm{M}}$ and MACHO $R_{\mathrm{M}}$ data, respectively. In the final fit we used the frequency corresponding to the highest detected peak among the different periodograms (see Table 2), but we cannot be certain that our choice is correct.

\section{3. $V 3=$ OGLE $051841.98-691051.9$}

The third star found to be an LMC $\beta$ Cephei star is about 2 mag brighter than V1 and V2 (Fig. 1) and shows a very complicated frequency pattern. As many as five frequencies with signal-to-noise $(S / N)$ ratio larger than 4 have been detected (see Sect. 3.5 for the definition of $S / N$ ). Figure 5 shows consecutive steps of prewhitening for the OGLE-II $I_{\mathrm{C}}$ data. One can see that all terms have very low amplitudes (Table 2). The last detected frequency is rather doubtful. Moreover, the frequency $2.0055 \mathrm{~d}^{-1}$ is very close to 2 (sidereal days) ${ }^{-1}$ which often appears in periodograms of bright stars. It is thus probably an artifact. However, the other low-frequency term, with $P \approx$ $0.5938 \mathrm{~d}$, can be real. According to the recent calculations of Pamyatnykh (1999), massive stars in this region of the H-R diagram have unstable modes of such long
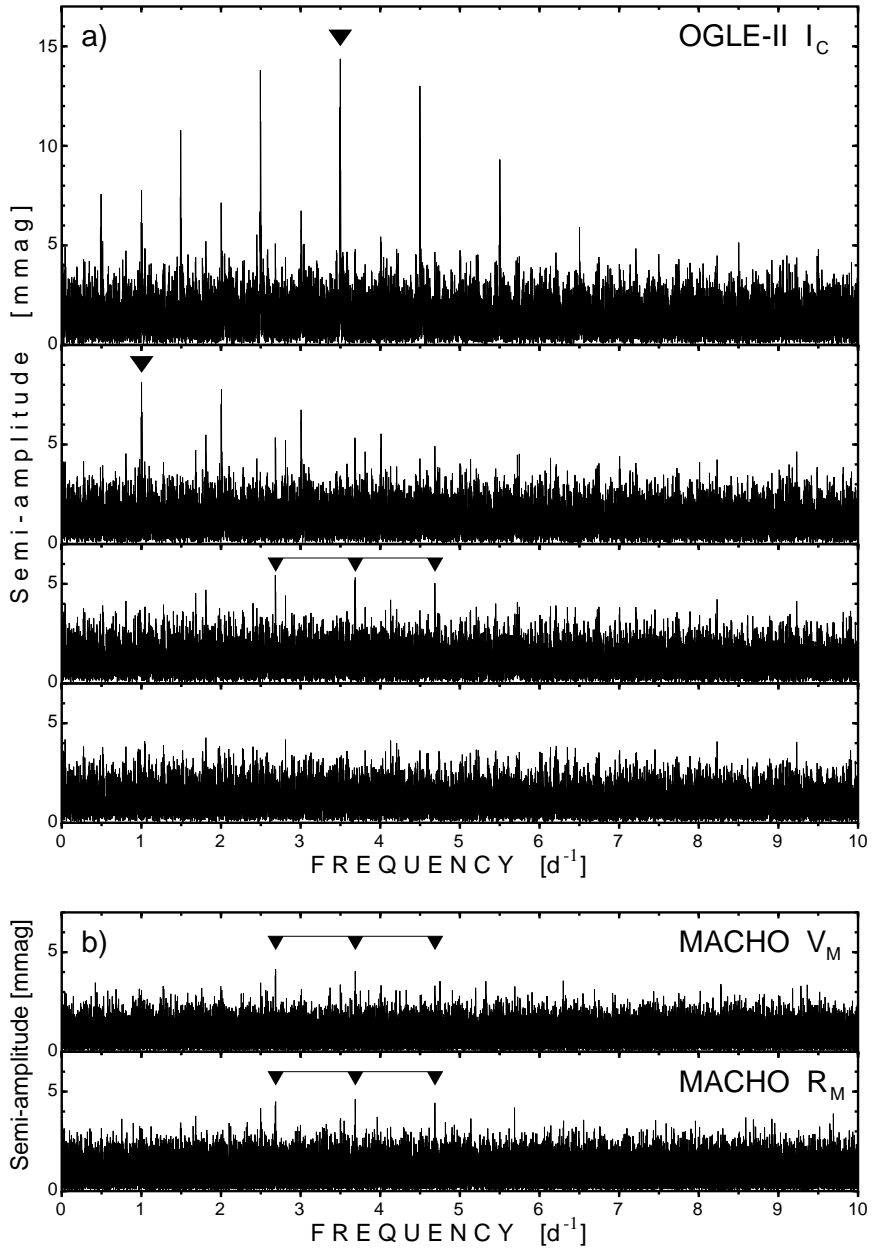

Fig. 4. a) Fourier periodograms of the OGLE-II 1997-2000 $I_{\mathrm{C}}$-filter observations of V2. From top to bottom the panels show the periodograms (i) of the original data, (ii) after prewhitening with $f_{1}=3.49767 \mathrm{~d}^{-1}$, (iii) after prewhitening with $f_{1}$ and $f_{\mathrm{sd}}=1.00333 \mathrm{~d}^{-1}$, (iv) after removing $f_{1}$, $f_{\text {sd }}$, and $f_{2}=3.68377 d^{-1}$. b) Fourier periodograms of the MACHO $V_{\mathrm{M}}$ (top) and $R_{\mathrm{M}}$ (bottom) data after prewhitening with the low-frequency variations and $f_{1}$. The triangles indicate the location of $f_{2}$ and its $1 \mathrm{~d}^{-1}$ aliases.

periods, thus the low-frequency variation may also represent pulsation.

Unfortunately, the star was not observed by the $\mathrm{MACHO}$ and we cannot verify the reality of the modes independently, as we did for V1 and V2. Also, there are not enough datapoints in the OGLE-II $B$ and $V$ band observations to detect reliably a similar variation. 
Table 2. The results of the sine curve fits to the photometric data of V1-V3. $N_{\text {obs }}$ is the number of individual observations and RSD is the residual standard deviation. See Sect. 3.5 for the definition of the signal-to-noise $(S / N)$ ratio.

\begin{tabular}{|c|c|c|c|c|c|c|c|c|c|}
\hline Star & $\begin{array}{l}\text { Frequency } \\
\qquad\left[\mathrm{d}^{-1}\right]\end{array}$ & $\begin{array}{l}\text { Period } \\
\text { [d] }\end{array}$ & $\begin{array}{c}\text { Data } \\
\text { source }\end{array}$ & Filter & $N_{\text {obs }}$ & $\begin{array}{r}\text { Semi-amplitude } \\
{[\mathrm{mmag}]}\end{array}$ & $\begin{array}{l}\text { HJD of the time } \\
\text { of maximum light }\end{array}$ & $\begin{array}{c}\text { RSD } \\
{[\mathrm{mmag}]}\end{array}$ & $S / N$ \\
\hline \multirow[t]{5}{*}{$\mathrm{V} 1$} & 4.051593 & 0.2468165 & OGLE-II & $B$ & 22 & $45.1 \pm 4.8$ & $51137.1511 \pm .0043$ & 15.5 & 7.8 \\
\hline & & & OGLE-II & $V$ & 45 & $37.7 \pm 3.1$ & $51058.4247 \pm .0036$ & 15.5 & 9.3 \\
\hline & & & OGLE-II & $I_{\mathrm{C}}$ & 350 & $38.9 \pm 1.0$ & $51027.8218 \pm .0010$ & 12.7 & 32.3 \\
\hline & & & MACHO & $V_{\mathrm{M}}$ & 816 & $39.7 \pm 0.9$ & $49947.2654 \pm .0009$ & 18.1 & 23.3 \\
\hline & & & MACHO & $R_{\mathrm{M}}$ & 767 & $37.2 \pm 0.9$ & $49896.1763 \pm .0010$ & 17.9 & 32.7 \\
\hline \multirow[t]{6}{*}{$\mathrm{V} 2$} & 3.497909 & 0.2858851 & OGLE-II & $I_{\mathrm{C}}$ & 501 & $12.4 \pm 1.1$ & $50837.3568 \pm .0041$ & 17.5 & 10.4 \\
\hline & & & MACHO & $V_{\mathrm{M}}$ & 1351 & $15.2 \pm 0.8$ & $49938.5438 \pm .0021$ & 18.9 & 16.3 \\
\hline & & & MACHO & $R_{\mathrm{M}}$ & 1188 & $13.6 \pm 0.8$ & $49915.6678 \pm .0027$ & 19.9 & 13.0 \\
\hline & 3.685343 & 0.2713452 & OGLE-II & $I_{\mathrm{C}}$ & 501 & $4.0 \pm 1.1$ & $50837.3874 \pm .0118$ & 17.5 & 3.3 \\
\hline & & & MACHO & $V_{\mathrm{M}}$ & 1351 & $3.6 \pm 0.7$ & $49938.6814 \pm .0089$ & 18.9 & 3.9 \\
\hline & & & MACHO & $R_{\mathrm{M}}$ & 1188 & $3.9 \pm 0.8$ & $49915.6335 \pm .0093$ & 19.9 & 3.7 \\
\hline \multirow[t]{5}{*}{ V3 } & 5.179046 & 0.1930858 & OGLE-II & $I_{\mathrm{C}}$ & 471 & $5.1 \pm 0.5$ & $50852.1238 \pm .0027$ & 6.8 & 9.3 \\
\hline & 3.495009 & 0.2861223 & OGLE-II & $I_{\mathrm{C}}$ & 471 & $3.9 \pm 0.4$ & $50852.0745 \pm .0054$ & 6.8 & 7.1 \\
\hline & 2.005502 & 0.4986283 & OGLE-II & $I_{\mathrm{C}}$ & 471 & $4.5 \pm 0.5$ & $50852.2471 \pm .0092$ & 6.8 & 8.2 \\
\hline & 1.684015 & 0.5938189 & OGLE-II & $I_{\mathrm{C}}$ & 471 & $3.1 \pm 0.4$ & $50852.1539 \pm .0137$ & 6.8 & 5.6 \\
\hline & 3.816132 & 0.2620454 & OGLE-II & $I_{\mathrm{C}}$ & 471 & $2.3 \pm 0.5$ & $50851.9626 \pm .0081$ & 6.8 & 4.1 \\
\hline
\end{tabular}

${ }^{a}$ The time is calculated to be as close as possible to the mean epoch of all observations of a given dataset. The first two digits of HJD, that is " 24 ", have been subtracted.

\subsection{Other short-period variables}

As mentioned in Sect. 2, two other short-period variables may also be $\beta$ Cephei-type stars. The first one, $V 4=$ OGLE $051652.03-691405.2=$ MACHO 79.5863.500 is located in the OGLE-II SC8 and MACHO 79 field. Its light curve is almost strictly sinusoidal in shape, has a period $P=0.260083 \mathrm{~d}$, and a semi-amplitude of about 0.10 mag in all three OGLE-II filters. There are two characteristics which are difficult to explain if we assume the star to be a $\beta$ Cephei variable. First, with $V=17.683$, $B-V=+0.066$, and $V-I_{\mathrm{C}}=-0.008$, the star falls in the colour-magnitude diagram about 1 mag below V1 and V2 (Fig. 1). Provided that V4 is not $\sim 20 \mathrm{kpc}$ behind the LMC - which is rather unlikely - this position corresponds to a mid-B-type star (Fig. 1), too cool to have low-degree $p$ modes excited. Second, for such a large amplitude, we would expect to see non-sinusoidal variations. For the two $\beta$ Cephei stars with the largest amplitudes known, BW Vul and $\sigma$ Sco, the light-curve shows the socalled "stillstand", a bump on the rising branch of the light curve (Sterken et al. 1986; Jerzykiewicz \& Sterken 1984). Since this is not observed, classifying V4 as a $\beta$ Cephei variable is open to doubt.
There is, however, an alternative explanation for the variability of this star. It could be a very rare contact or nearly contact binary consisting of two similar mid-B-type stars and seen at low inclination. Simple calculations indicate that with $2 P \approx 0.52 \mathrm{~d}$ we can have a contact binary containing two main-sequence stars with masses of 5-6 $M_{\odot}$. Such an object would have the position of V4 in Fig. 1.

The next star we suspected to be a $\beta$ Cephei variable, V5 = OGLE053811.81-704245.7 = MACHO11.9350.116, is very peculiar. It is found in the OGLE-II SC17 and MACHO 11 field. The star is fainter than V1 and V2 $(V=$ 17.509), and has much redder colours $(B-V=+0.232$, $\left.V-I_{\mathrm{C}}=+0.351\right)$. However, it is located very close to the LMC nebula and cluster NGC $2075=\mathrm{N} 213$ and in addition the SC17 field has a large reddening spread (see the colour-magnitude diagram presented by Udalski et al. 2000). Consequently, its colours could be accounted for by the reddening effect which will move the star, along the reddening line, close to V1 and V2.

If the star were a $\beta$ Cephei variable, it would be indeed an unusual object. While the two detected frequencies $\left(f_{1}=3.780 \mathrm{~d}^{-1}, f_{2}=3.777 \mathrm{~d}^{-1}\right)$ are typical for a $\beta$ Cephei star, the amplitudes are enormous. The amplitude of the $f_{1}$ term in $B$ reaches $0.43 \mathrm{mag}$, exceeding about 




Fig. 5. Fourier periodograms of the OGLE-II 1997-2000 $I_{\mathrm{C}}$-filter observations of V3. From top to bottom the panels show consecutive steps of prewhitening with frequencies (given in Table 2) indicated by triangles.

twice the amplitude in BW Vul. There is also a strong dependence of the amplitude on wavelength: in the $I_{\mathrm{C}}$ the amplitude is about half that in $B$. The second mode has smaller amplitudes, but still rather large for a $\beta$ Cephei star, $0.24 \mathrm{mag}$ in $B$ and $0.10 \mathrm{mag}$ in $I_{\mathrm{C}}$. The two frequencies are very close to each other and the beating with a period longer than 300 days is clearly seen in the data (Fig. 6). We also found that both periods increase. The rate of change of $P_{2}=f_{2}^{-1}$ amounts to about $1.7 \times 10^{-8}$ and is seven times larger than that of $P_{1}$. As a result of the changes of the periods, the beat period shortened from $380 \mathrm{~d}$ in 1993 to about $317 \mathrm{~d}$ in 2000.

Recalling the arguments given above for V4, we see that almost sinusoidal variations observed in V5 with even larger amplitudes than in V4 are difficult to reconcile with $\beta$ Cephei-type variability. There is, however, another possibility. The star can be an RR Lyrae variable of Bailey type c. The colours, periods, amplitudes and the shape of the light curve agree well with this type of variability. Moreover, RRc stars with very close periods have been recently found (Olech et al. 1999a, 1999b; Moskalik 2000). In the new classification scheme of Alcock et al. (2000), the star would be of RR1- $v 1$ type. However, V5 would then be too bright to be an LMC star. In accordance with its $V$ magnitude, the star would be a foreground object located approximately half way to the LMC. There is another, indirect argument in favour of this explanation: RR Lyraes, especially of the RRc type, are known to exhibit large erratic period changes (see, e.g.,

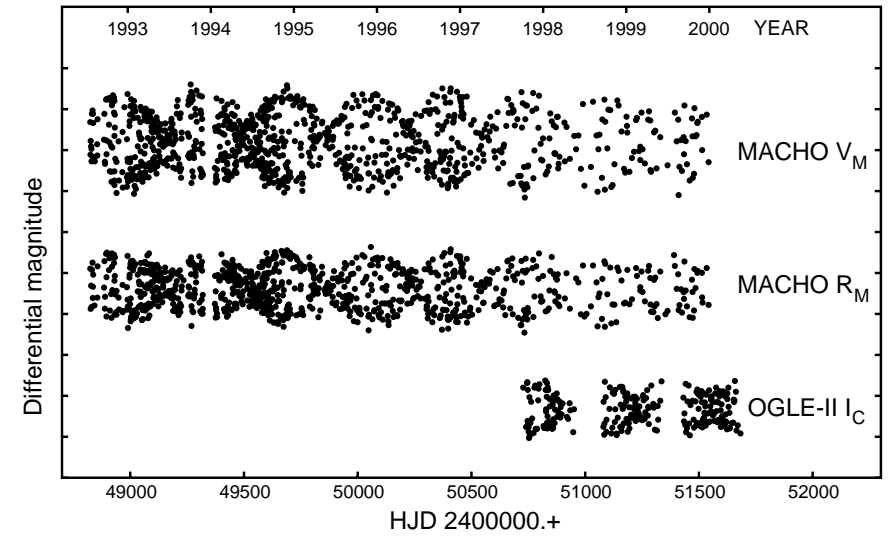

Fig. 6. The OGLE-II $I_{\mathrm{C}}$ and MACHO $V_{\mathrm{M}}$ and $R_{\mathrm{M}}$ light curves of V5. Note the beating due to the presence of two periodicities with very close frequencies. Ordinate ticks are separated by 0.2 mag.

Jurcsik et al. 2001; Kopacki 2001) which are rather not observed in the $\beta$ Cephei stars (Jerzykiewicz 1999). In any case, the star is very unusual and deserves further attention.

As far as V5 is concerned, the low-resolution spectroscopy would be sufficient to distinguish between the two possibilities. Even accurate Johnson $U$ photometry, which is probably soon going to become available (Zaritsky et al. 1997) would be conclusive in this case. For V4 we need radial velocities to decide on the final classification.

\subsection{Detection threshold}

In view of the results presented here and the discussion that follows in Sect. 6, it is useful to estimate the detection threshold of the search. We therefore analyzed the light curves of 36 variables randomly selected from the catalogue of Żebruń et al. (2001b) with $14.5<V<19.1$ mag. First, all periodic signals were removed from the data. Next, Fourier periodograms of the residuals were calculated giving mean semi-amplitudes between 0 and $10 \mathrm{~d}^{-1}$. We define this value as the noise $(N)$. It is easy to find that if we define signal $(S)$ as the height of the maximum peak in the Fourier periodogram, the signal-tonoise ratio $(S / N)$ for a pure Gaussian noise would be $(S / N)_{\text {noise }} \approx 3.0$. Therefore, we have arbitrarily set our detection threshold to $D_{\text {th }}=4 N$. The thresholds defined in this way are shown in Fig. 7 for the stars mentioned above. It can be seen from the figure that in the range of magnitudes where the LMC $\beta$ Cephei stars are expected to occur, we should be able to detect amplitudes as low as 3-5 mmag. This agrees very well with the results given in Sects. 3.1-3.3 and Table 2. Since the OGLE-II DIA photometry practically does not suffer from crowding, the relation shown in Fig. 7 should apply to almost all variables.

However, we have to remember that the analysis was carried out only on those stars, already found as variables by the DIA. In order to check whether the DIA missed some small-amplitude variables which could be detected by periodogram analysis, we calculated $S / N$ for all 


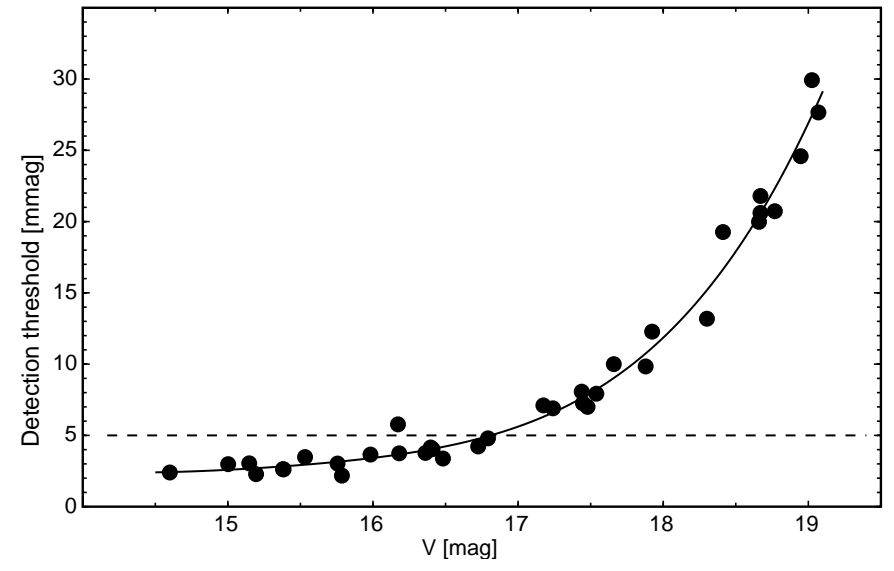

Fig. 7. The detection threshold, $D_{\mathrm{th}}$, for the OGLE-II DIA $I_{\mathrm{C}^{-}}$ filter photometry of 36 stars (dots) in the LMC as a function of the $V$ magnitude. The continuous line is the polynomial fit to the points, the dashed line shows the detection threshold of $5 \mathrm{mmag}$. See text for the definition of $D_{\mathrm{th}}$.

candidate variables in the SC1 field using their Fourier periodograms in the range between 0 and $10 \mathrm{~d}^{-1}$. Prior to analysis, we additionally filtered the observations using a $4 \sigma$ clipping in order to avoid the influence of outliers. The $S / N$ values calculated in this way have a very well defined limit of $(S / N) \approx 3.6$, independent of the magnitude. This shows that the DIA did not miss low-amplitude variables and its detection threshold is practically the same as that shown in Fig. 7.

It is also interesting to compare the accuracy of the OGLE-II and MACHO data. As can be seen from the comparison of the RSD values in Table 2, the accuracy of a single observation is slightly better in the OGLE-II data. However, because there are usually more observations for a given star in the MACHO database, the noise levels in the periodograms are similar for both data sets.

\section{Relation with clusters and associations}

The $\beta$ Cephei-type variability is confined to stars in the core-hydrogen burning phase of evolution or shortly beyond this phase having masses larger than $\sim 7 M_{\odot}$ (see, e.g., Pamyatnykh 1999). From the comparison with evolutionary models it can easily be shown that a $\beta$ Cephei star cannot be older than $\sim 35 \mathrm{Myr}$. This is why almost all Galactic $\beta$ Cephei stars can be identified as the members of the young open clusters or OB associations. We therefore expect that the LMC $\beta$ Cephei-type stars can also be members of clusters or associations.

\subsection{V1 and the LH81 association}

Our first variable, V1, is located in the middle of the ring-shaped superbubble N 154 (Henize 1956) = DEM 246 (Davies et al. 1976) which is also a diffuse X-ray source (Dunne et al. 2001). The two brightest parts of N 154, south-western BSDL 2434 (Bica et al. 1999) and the much brighter north-eastern LH 87n, with a bright knot NGC 2048, are well seen in Fig. 8. N 154 encompasses two large OB associations: LH 81 (Lucke \& Hodge 1970) = SL 589 (Shapley \& Lindsay 1963) and LH 87 (see Fig. 8). Several compact groups within LH 81 are usually distinguished as open clusters; the brightest one is the OGLECL-LMC605 (Pietrzyński et al. 1999) = BCDSP 8 (Bica et al. 1996). The LH 81 association has been studied by Massey et al. (2000) and is known to contain many very hot and massive objects including three Wolf-Rayet stars and two stars of spectral type O3-4. Figure 9 shows the colour-magnitude diagram for a region in $\mathrm{LH} 81$ with a radius of 2.7 centered at $(\alpha, \delta)_{2000.0}=\left(5^{\mathrm{h}} 34^{\mathrm{m}} 39^{\mathrm{s}}\right.$, $\left.-69^{\circ} 43^{\prime} 11^{\prime \prime}\right)$. We see that the main sequence extends to stars as bright as eleventh magnitude and that V1 is a likely member of the association. If this is true and the star formation was coeval, V1 should not be older than $5 \mathrm{Myr}$, the age of LH 81 deduced by Massey et al. (2000).

\subsection{V2 and the LH59 association}

$\mathrm{V} 2$ is located about $7^{\prime}$ north-east from the center of the LH 59 association (Fig. 8). The nearest clusters are the OGLE-CL-LMC499 = BSDL 1861 and OGLE-CLLMC505 = BSDL 1892. The colour-magnitude diagrams of the clusters provided by Pietrzyński et al. (1999) show that at least BSDL 1892 contains some hot stars. The ages of the two clusters estimated by Pietrzyński \& Udalski (2000) are 80 and 250 Myr for BSDL 1861 and 1892 , respectively. Thus, the clusters are much too old to contain $\beta$ Cephei stars. However, LH 59 seems to be slightly younger. Dieball \& Grebel (2000) estimated the age of the three rich clusters embedded in this association (NGC 1969, 1971, 1972) to be in the range 40-70 Myr. Taking into account the errors in the age estimates and the possibility that the formation was not strictly coeval in such a large complex, the presence of a $\beta$ Cephei star in this region might still be possible. Although we cannot be certain that V2 belongs to LH 59, the density of hot stars in this region is quite high. We conclude that V2 belongs either to the coronal region of LH 59 or to the LMC field, where, as found e.g. by Massey et al. (1995) and Holtzman et al. (1999), the star formation is still in progress.

We also note that V2 has been detected as a farultraviolet source by the UIT telescope. Its B1-filter magnitude of $14.07 \pm 0.07$ (Parker et al. 1998) indicate that it is really an early B-type star.

\subsection{V3 and the LH41 association}

It is, in turn, easy to indicate a connection of our third variable, V3, with an LMC association. The star is located in the rich LH 41 association known to contain S Doradus, the extreme Luminous Blue Variable (LBV, Humphreys \& Davidson 1994). The association (Fig. 8) contains also another probable LBV star, R 85, a Wolf-Rayet star, Brey 21, and many O-type stars (Breysacher et al. 1999; 

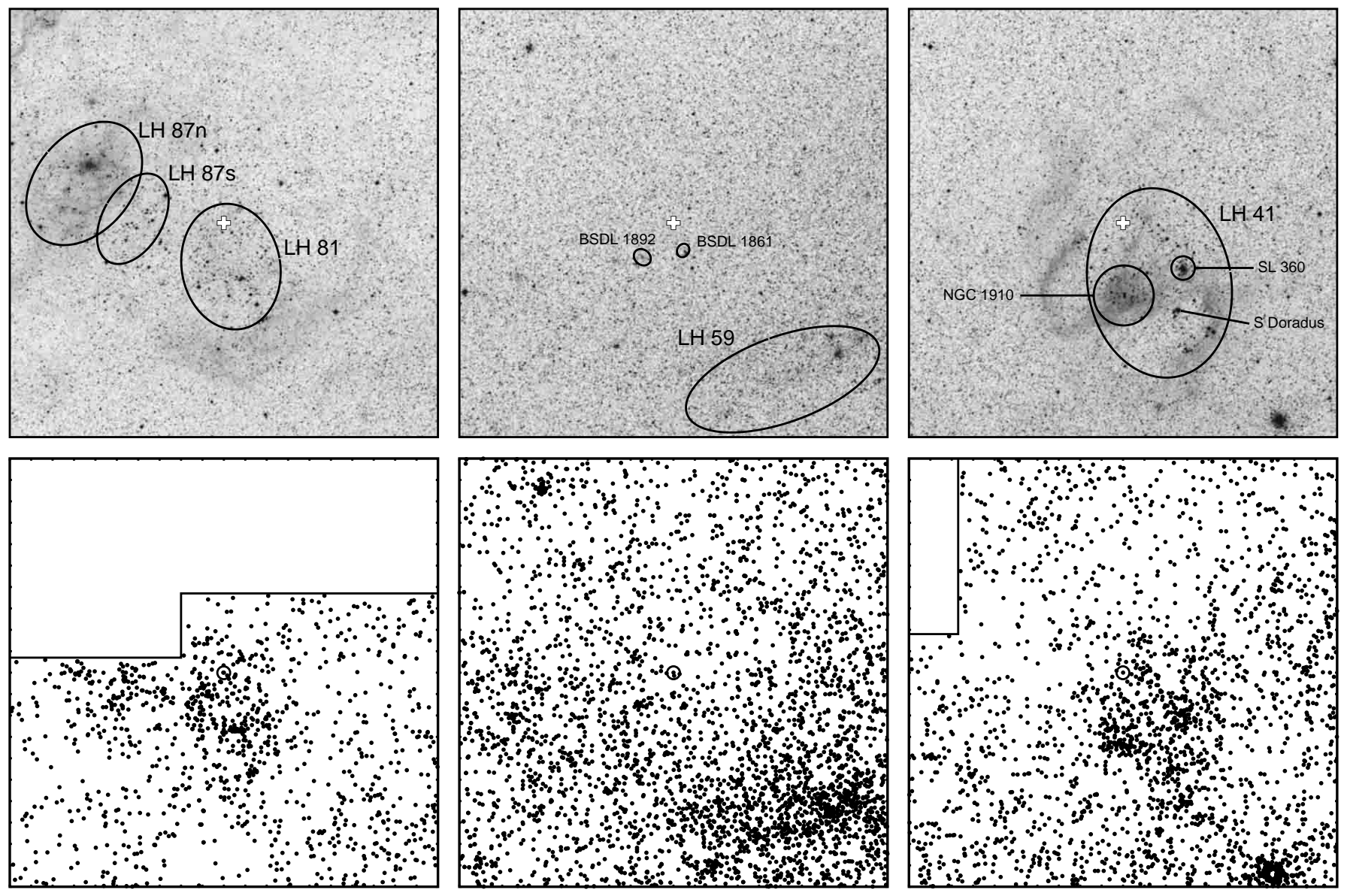

Fig. 8. Three $20^{\prime} \times 20^{\prime}$ fragments of the red-filter POSS-II plates centered at V1 (left), V2 (middle), and V3 (right). Associations, clusters and stars discussed in the text are labeled. White crosses mark the positions of $\beta$ Cephei variables. Below, for each variable (encircled) the location of nearby bright, hot stars with $V<18$ mag and $\left(V-I_{\mathrm{C}}\right)<0.5$ mag, taken from the catalogue of Udalski et al. (2000), is shown on the same scale. North is up, east to the left.
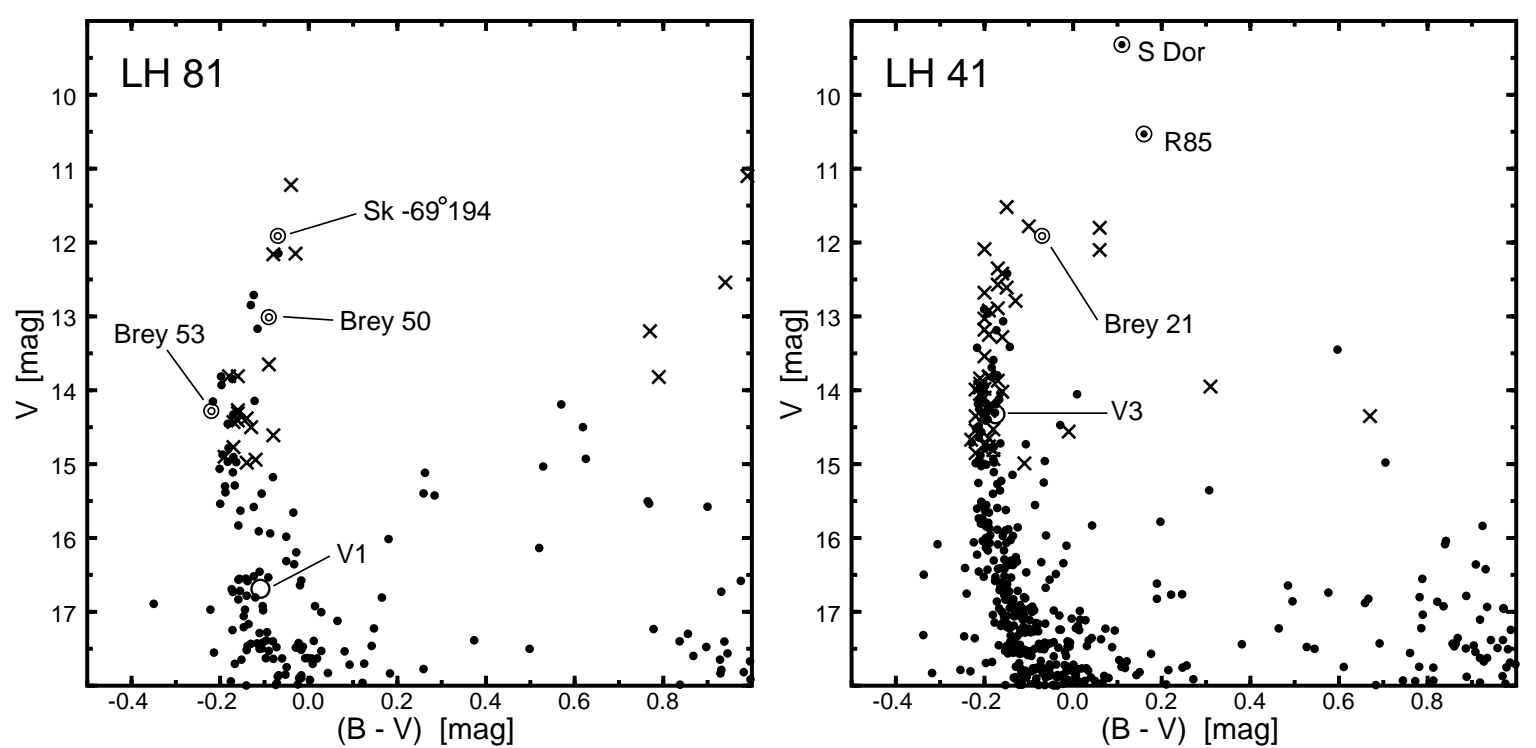

Fig. 9. Colour-magnitude diagrams for the brightest stars of the LH 81 (left) and LH 41 (right) association. The photometry of stars shown as dots was taken from Udalski et al. (2000), those shown as crosses, from Massey et al. (2000). The Wolf-Rayet stars are shown as encircled open circles, LBVs, as encircled dots and $\beta$ Cephei stars, as open circles, in addition to a label. Only stars with $(B-V)<1.0 \mathrm{mag}$ and $V<18$ mag are shown in the plots. 
Massey et al. 2000). Several young clusters including the compact SL $360=$ HD 35342 and the larger NGC $1910=$ SL 371, form the well-seen groups within the association. The whole region is surrounded by the N $119=$ DEM 132 nebula (Fig. 8). There is no doubt that intensive star formation is taking place here. The age of the association estimated by Massey et al. (2000) is 4 Myr. The colourmagnitude diagram for the region of LH 41 with a 4.5 radius centered on $(\alpha, \delta)_{2000.0}=\left(5^{\mathrm{h}} 18^{\mathrm{m}} 30^{\mathrm{s}},-69^{\circ} 13^{\prime} 15^{\prime \prime}\right)$ is shown in Fig. 9.

The $U B V$ photometry of $\mathrm{V} 3$ has been obtained by Massey et al. (2000). The star has been designated as LH41-64 by these authors and has $V=14.32, B-V=$ -0.16 and $U-B=-1.02 \mathrm{mag}$. Thus we get $E(B-V)=$ $0.14 \mathrm{mag}$ and intrinsic colours: $(B-V)_{0}=-0.30$ and $(U-B)_{0}=-1.12 \mathrm{mag}$ which correspond rougly to a B0/O9 type star.

\section{Notes on previous searches}

As mentioned in the Introduction, the previous searches for $\beta$ Cephei stars in the LMC (Sterken \& Jerzykiewicz 1988; Kubiak 1990; Balona 1993) yielded some variables, although none of them could be convincingly classified as a $\beta$ Cephei variable. With the OGLE-II and MACHO photometry at hand we can now try to verify their variability.

Out of the six program and five comparison stars from Brunet et al. (1975) selected for observations by Sterken \& Jerzykiewicz (1988), only star 90 and 134 are within the OGLE-II fields. Both stars were identified by the DIA as variables. However, the amplitudes are very small (less than 0.006 mag for star 90 and 0.01 mag for star 134) and are very likely of instrumental origin. On the other hand, the MACHO observed all these stars but 230. From our analysis of the ten stars we found that: (i) none of the stars shows short-period variability, (ii) stars 32 and 257 are variable, but the light changes are aperiodic and have the amplitudes below $0.2 \mathrm{mag}$, (iii) a 1-year periodicity is seen for four stars $(90,146,230$, and 134), but comparing the OGLE-II photometry of stars 90 and 134 we are certain that the variations are of instrumental origin, (iv) stars 269 and 271 are heavily blended. Concluding, none of the stars show $\beta$ Cephei-type variability.

Kubiak (1990) found nine variables in the LMC cluster NGC 1712. Of these, seven (all but 5 and 8) are included in the MACHO field 44. Variable 6 is a blend and we could not cross-identify it properly, but the three nearby MACHO stars are constant. Of the remaining six stars, only variable 1 seems to be constant. Variable 2 shows a smooth increase and then decrease of brightness with an amplitude of $0.4 \mathrm{mag}$ in $V_{\mathrm{M}}$ and $0.5 \mathrm{mag}$ in $R_{\mathrm{M}}$. Variables 3 and 4 are eclipsing binaries with Algol-type light curves. The eclipses of equal depth (0.42 mag for variable 3 and $0.17 \mathrm{mag}$ for variable 4 ) indicate that the binaries have equal components. Variable 7 has a small amplitude and a probable period of $0.94 \mathrm{~d}$, while variable 9 shows irregular variations mainly in the $V_{\mathrm{M}}$ band.
Table 3. Stars found to be variable in previous searches for $\beta$ Cephei stars in the LMC identified with MACHO sources.

\begin{tabular}{rcl}
\hline \hline Star & MACHO name & Comment on variability \\
\hline \multicolumn{3}{c}{ Sterken \& Jerzykiewicz $(1988)$} \\
\hline 32 & 18.2479 .10 & Irregular variations \\
90 & 79.4774 .10 & Constant \\
216 & 49.6736 .14 & Constant \\
257 & 8.8784 .291 & Constant \\
\hline \multicolumn{3}{c}{ Kubiak (1990) } \\
\hline 1 & 44.1626 .20 & Constant. \\
2 & 44.1626 .35 & Long-period variable \\
3 & 44.1626 .46 & Eclipsing, $P=2.291265 \mathrm{~d}$ \\
4 & 44.1626 .46 & Eclipsing, $P=1.636929 \mathrm{~d}$ \\
7 & 44.1747 .28 & $P=0.9403 \mathrm{~d} ?$ \\
9 & 44.1868 .9 & Irregular \\
\hline \multicolumn{3}{c}{ Balona (1993) } \\
\hline 87 & 61.8192 .120 & Eclipsing, $P=1.662846 \mathrm{~d}$ \\
241 & 61.8192 .154 & Irregular variations \\
297 & 61.8192 .55 & Constant \\
491 & 61.8191 .12 & Constant \\
682 & 61.8191 .81 & Constant \\
\hline
\end{tabular}

Balona (1993) detected variability of seven stars in another LMC cluster, NGC 2004. Of those, five have photometry in the MACHO database. We confirm the variability of only two (Table 3). Star 87 is an eclipsing binary with a $\beta$ Lyrae-type light curve and an orbital period of $1.662846 \mathrm{~d}$ while star 241 is an irregular variable. The period of 1.835 d given by Balona (1993) for the latter star is not confirmed. Stars 297, 491, and 682 were found to be constant.

The other LMC cluster searched by Balona (1993), NGC 2100, was not observed by either the MACHO or the OGLE-II.

\section{Discussion}

$\beta$ Cephei-type stars have been finally found in the LMC. These are the first extragalactic stars of this type so far discovered. Photometric indices, magnitudes, amplitudes and periods of three variables fit the $\beta$ Cephei characteristics. However, the spectroscopic confirmation and/or the spectral type of these variables would be, of course, desirable.

Since there are over 27000 early B-type stars in the LMC area covered by the OGLE-II observations, and the variability detection threshold was rather low, we may conclude that for the LMC the BCIS has been mapped. Only three $\beta$ Cephei-type variables have been found. Two important facts arise from this finding. The first is the fraction of $\beta$ Cephei stars with respect to the whole population of early B-type stars, the other is their position in the colour-magnitude diagram. 


\subsection{Fraction of $\beta$ Cephei stars with respect to early $B$-type stars in the $L M C$}

Practically all Galactic $\beta$ Cephei stars have $M_{V}$ between -4.5 and $-1.5 \mathrm{mag}$. We take this range as a reference and in order to avoid selection effects we restrict the comparison to stars in open clusters. Moreover, to make the comparison reliable, we need to account for different detection thresholds. A detailed analysis of the fraction of $\beta$ Cephei stars in Galactic open clusters is going to be published soon (Pigulski et al., in preparation), here we mention only the preliminary result presented by Pigulski et al. (2002). These authors pointed out that the fraction of $\beta$ Cephei stars in Galactic southern clusters is few times larger than in the northern ones, and, if related to all main-sequence stars falling into the $M_{V}$ range given above it amounts to $35 \pm 7 \%$ for the southern and $5.3 \pm 1.7 \%$ for the northern clusters. Since southern clusters are closer to the Galactic centre, this was explained as being a consequence of the Galactic metallicity gradient. At the LMC distance, $-4.5 \leq M_{V} \leq-1.5 \mathrm{mag}$ corresponds roughly to $14.3 \leq V \leq 17.3 \mathrm{mag}$. There are 27663 main-sequence stars in the OGLE-II fields falling in this range of $V$ magnitude. (As a main-sequence star we mean here a star with $\left(V-I_{\mathrm{C}}\right)<0.5 \mathrm{mag}$; since contamination by foreground stars is very low in this part of the colour-magnitude diagram, it does not affect our estimate.) The three $\beta$ Cephei stars we found, constitute $3 / 27663 \approx 0.011 \pm 0.006 \%$ of early B-type stars (we assume a Poisson statistics to derive errors). Even if we take into account the fact that for searches in the Galactic open clusters roughly twice as low threshold is achieved as that seen in Fig. 7, so that some small-amplitude variables could have been missed in the LMC, the striking difference in the $\beta$ Cephei fraction between the Galaxy and the LMC is evident. In other words, as the average LMC metallicity is $[\mathrm{M} / \mathrm{H}] \approx-0.4$ (or $Z \approx 0.007$ ), this is another observational confirmation of the fact that the driving mechanism strongly depends on the metallicity.

\subsection{Position of $\beta$ Cephei-type stars in the colour-magnitude diagram: A comparison with theoretical predictions for LMC metallicity}

Should we, however, expect to observe $\beta$ Cephei stars at all in the LMC if $Z=0.007$ ? The two recent theoretical predictions (Pamyatnykh 1999; Deng \& Xiong 2001) give different answers to this question. Pamyatnykh (1999, see his Fig. 11) for $Z=0.01$ does not find unstable modes for stars with masses smaller than $25 M_{\odot}$. On the other hand, Deng \& Xiong (2001) predict instability for stars with masses $\sim 10 M_{\odot}$ down to $Z=0.005$. The following reasons coming from our LMC results favour the predictions of Pamyatnykh (1999):

- First, we find $\beta$ Cephei stars both among low mass (8$10 M_{\odot}, \mathrm{V} 1$ and $\left.\mathrm{V} 2\right)$ and high mass $\left(25-30 M_{\odot}, \mathrm{V} 3\right)$ stars. In Fig. 11 of Pamyatnykh (1999), the BCIS is shown for three values of $Z: 0.01,0.015$ and 0.02 . We see in this figure that for $Z=0.015$ the lower part of the BCIS covers about half of the main-sequence width. Going towards higher masses the BCIS becomes narrower and then widens again. For $Z=0.01$ there is no lower part at all and instability begins for masses $\sim 25 M_{\odot}$. It seems that for a value of $Z$ between 0.01 and 0.015 the BCIS splits in two regions, one for masses around $10 M_{\odot}$ and one over $25 M_{\odot}$. This does correspond to the situation observed in the LMC;

- In order to obtain the agreement mentioned above, we need, however, to have either a higher metallicity in the LMC $\beta$ Cephei stars or the splitting of the BCIS described above, but for $Z=0.007$. Although Pamyatnykh (1999), comparing the location of the hot border of the BCIS based on OP and OPAL opacities, estimates that an error in $Z$ of about $0.003-$ 0.005 can be involved, we think that the first possibility is more probable. We know from Sect. 4 that at least two, and perhaps all three $\beta$ Cephei stars in the LMC are located in regions of violent star formation. The recent studies of the age-metallicity relation for the LMC (Cohen 1982; Pagel \& Tautvaišienè 1998) and direct metallicity determinations in young objects (Olszewski et al. 1991; Luck \& Lambert 1992; Jasniewicz \& Thévenin 1994; Rolleston et al. 1996; Luck et al. 1998; Korn et al. 2000) indicate that although the mean LMC metalicity is about $[\mathrm{M} / \mathrm{H}] \approx$ -0.4 , a large spread in metallicities of young objects is observed. It is even possible that some objects have solar or nearly solar metallicity. In fact, it would be naïve to believe that dozen megayears of the LMC history did not leave any metallicity inhomogeinities. It is therefore very likely that our variables have a metallicity higher than the LMC average. Nevertheless, spectroscopic determination of abundances in these stars would be highly desirable;

- Why do we think the predictions of Deng \& Xiong (2001) are incorrect? First, these authors do not find the instability among massive stars, so it would be difficult to explain the variability of V3 with a lower than Galactic metallicity. Next, with instabilities predicted down to $Z=0.005$, we should have found many more $\beta$ Cephei stars than we did. Finally, Deng \& Xiong (2001) used the first 1992 version of the OPAL opacity tables with an analytical approximation which-as these authors write-gives as much as $10 \%$ differences with respect to the tabulated values. In view of the subtle domination of driving over damping, this could influence considerably the final result.

\section{Summary and future work}

The most important results obtained in this paper can be summarized as follows:

1. Three $\beta$ Cephei stars have been found in the LMC bar. 
2. Two of them were found to have $V$ magnitude corresponding roughly to the LMC B2-B2.5 stars, while the third one (V3) is as bright as a B0-type star.

3. The three variables constitute a very small fraction (less than $0.02 \%$ ) of all early B-type stars falling within the range of magnitudes where the Galactic stars of this type are distributed. This is much less than for the Galactic clusters and can be explained by a strong dependence of the driving mechanism on metallicity.

4. The location and number of $\beta$ Cephei variables in the $\mathrm{LMC}$ is in reasonable agreement with the theoretical predictions of Pamyatnykh (1999) provided that the metallicity of these stars is slightly higher than the LMC average for young population, namely, between $Z=0.01$ and $0.015([\mathrm{M} / \mathrm{H}]$ between -0.3 and -0.1$)$.

5. All three variables were found to be in or very close to regions of intensive star formation, implying that they indeed can have enhanced metallicities.

6. If the low-frequency variation in V3 is confirmed, this can be an interesting example of a luminous $\beta$ Cephei star in which modes with longer than typical periods are excited. The star could be therefore a good target for asteroseismology.

The main goal of this series of papers is to give a thorough observational picture of the variability among the LMC and SMC stars lying in the upper main sequence, where the $\kappa$ mechanism related to the metallicity bump is at work. For this purpose we shall use, like in the present paper, the OGLE-II and MACHO photometric databases. In particular, we are going to focus on the study of slowly pulsating B stars (SPB), which, although fainter than $\beta$ Cephei variables, have typical amplitudes large enough to be detected in the LMC and even in the SMC. Next, we are going to search bright B and A-type stars for variability with periods of the order of 1 day and longer. This would probably shed some light on the presence of the $g$ modes in luminous early-type stars. Finally, the variability of Be stars and hot eclipsing binaries will be studied as well.

Acknowledgements. The paper has been supported by the KBN grant No. 2 P03D 006 19. We thank Prof. M. Jerzykiewicz, Drs. G. Kopacki and A. Pamyatnykh for fruitful discussions. The detailed comments of the anonymous referee are acknowledged. We also thank the staff of the Institute of Mathematics of our University, especially Mrs. M. Kołaczkowska, for making available their computing facilities.

This paper utilizes public domain data originally obtained by the MACHO Project, whose work was performed under the joint auspices of the U.S. Department of Energy, National Nuclear Security Administration by the University of California, Lawrence Livermore National Laboratory under contract No. W-7405-Eng-48, the National Science Foundation through the Center for Particle Astrophysics of the University of California under cooperative agreement AST-8809616, and the Mount Stromlo and Siding Spring Observatory, part of the Australian National University.

\section{References}

Alcock, C., Allsman, R. A., Alves, D. R., et al. 2000, ApJ 542, 257

Allsman, R. A., \& Axelrod, T. S. 2001 [astro-ph/0108444]

Balona, L. A. 1992, MNRAS, 256, 425

Balona, L. A. 1993, MNRAS, 260, 795

Balona, L. A., \& Jerzykiewicz, M. 1993, MNRAS, 260, 782

Bica, E., Claría, J. J., Dottori, H., Santos, Jr. J. F. C., \& Piatti, A. E. 1996, ApJS, 102, 57

Bica, E. L. D., Schmitt, H. R., Dutra, C. M., \& Oliveira, H. L. 1999, AJ, 117, 238

Breysacher, J., Azzopardi, M., \& Testor, G. 1999, A\&AS, 137, 117

Brunet, J. P., Imbert, M., Martin, N., et al. 1975, A\&AS, 21, 109

Cohen, J. G. 1982, ApJ, 258, 143

Cook, K. H. 2002, private communication

Cox, A. N., Morgan, S. M., Rogers, F. J., \& Iglesias, C. A. 1992, ApJ, 393, 272

Davies, R. D., Elliot, K. H., \& Meaburn, J. 1976, MmRAS, 81, 89

Deng, L., \& Xiong, D. R. 2001, MNRAS, 327, 881

Dieball, A., \& Grebel, E. K. 2000, A\&A, 358, 897

Dunne, B. C., Points, S. D., \& Chu, Y.-H. 2001, ApJS, 136, 119

Dziembowski, W. A., \& Pamyatnykh, A. A. 1993, MNRAS, 262,204

Gautschy, A., \& Saio, H. 1993, MNRAS, 262, 213

Henize, K. G. 1956, ApJS, 2, 315

Holtzman, J. A., Gallagher III, J. S., Cole, A. A., et al. 1999, AJ, 118, 2262

Humphreys, R. M., \& Davidson, K. 1994, PASP, 106, 1025

Jasniewicz, G., \& Thévenin, F. 1994, A\&A, 282, 717

Jerzykiewicz, M. 1999, New Astron. Rev., 43, 455

Jerzykiewicz, M., \& Sterken, C. 1984, MNRAS, 211, 297

Jurcsik, J., Clement, C., Geyer, E. H., \& Domsa, I. 2001, AJ, 121,951

Kiriakidis, M., El Eid, M. F., \& Glatzel, W. 1992, MNRAS, $255,1 \mathrm{P}$

Kjeldsen, H., \& Baade, D. 1994, Proc. IAU Symp. 162, ed. L. A. Balona, H. F. Henrichs, \& J. M. le Contel, 29

Kopacki, G. 2001, Ph.D. Thesis, Wrocław University

Korn, A. J., Becker, S. R., Gummersbach, C. A., \& Wolf, B. 2000, A\&A, 353, 655

Krzesiński, J., \& Pigulski, A. 1997, A\&A, 325, 987

Kubiak, M. 1990, Acta Astron., 40, 297

Luck, R. E., \& Lambert, D. L. 1992, ApJS, 79, 303

Luck, R. E., Moffett, T. J., Barnes III, T. G., \& Gieren, W. P. 1998, AJ, 115, 605

Lucke, P. G., \& Hodge, P. W. 1970, AJ, 75, 171

Massey, P., Lang, C. C., DeGioia-Eastwood, K., \& Garmany, C. D. 1995, ApJ, 438, 188

Massey, P., Waterhouse, E., \& DeGioia-Eastwood, K. 2000, AJ, 119,2214

Moskalik, P. 2000, PASPC, 203, 315

Moskalik, P., \& Dziembowski, W. A. 1992, A\&A, 256, L5

Olech, A., Kałużny, J., Thompson, I. B., et al. 1999a, AJ, 118, 442

Olech, A., Woźniak, P. R., Alard, C., et al. 1999b, MNRAS, 310,759

Olszewski, E. W., Schommer, R. A., Suntzeff, N. B., et al. 1991, AJ, 101, 515

Pagel, B. E. J., \& Tautvaišienè, G. 1998, MNRAS, 299, 535 
Pamyatnykh, A. A. 1999, Acta Astron., 49, 119

Parker, J. W., Hill, J. K., Cornett, R. H., et al. 1998, AJ, 116, 180

Pietrzyński, G., \& Udalski, A. 2000, Acta Astron., 50, 337

Pietrzyński, G., Udalski, A., Kubiak, M., et al. 1999, Acta Astron., 49, 521

Pigulski, A., Kopacki, G., Kołaczkowski, Z., \& Jerzykiewicz, M. 2002, PASPC, 259, 146

Rolleston, W. R. J., Brown, P. J. F., Dufton, P. L., \& Howarth, I. D. 1999, A\&A, 315, 95

Schwarzenberg-Czerny, A. 1996, ApJ, 460, L107

Shapley, H., \& Lindsay, E. M. 1963, Irish AJ, 6, 74

Stanek, K. Z. 1996, ApJ, 460, L37

Sterken, C., \& Jerzykiewicz, M. 1988, MNRAS, 235, 565

Sterken, C., \& Jerzykiewicz, M. 1993, Sp. Sci. Rev., 62, 95
Sterken, C., Snowden, M., Africano, J., et al. 1986, A\&AS, 66, 11

Udalski, A., Kubiak, M., \& Szymański, M. 1997, Acta Astron., 47,319

Udalski, A., Szymański, M., Kubiak, M., et al. 1998, Acta Astron., 48, 147

Udalski, A., Szymański, M., Kubiak, M., et al. 2000, Acta Astron., 50, 307

Woźniak, P. R. 2000, Acta Astron., 50, 421

Zaritsky, D. 1999, AJ, 118, 2824

Zaritsky, D., Harris, J., \& Thompson, I. 1997, AJ, 114, 1002

Żebruń, K., Soszyński, I., \& Woźniak, P. R. 2001a, Acta Astron., 51, 303

Żebruń, K., Soszyński, I., Woźniak, P. R., et al. 2001b, Acta Astron., 51, 317 